\title{
CORRESPONDENCE RE: GUILLOU L, GEBHARD S, SALMERON M, COINDRE JM. METASTASIZING FIBROUS HISTIOCYTOMA OF THE SKIN: A CLINICOPATHOLOGIC AND IMMUNOHISTOCHEMICAL ANALYSIS OF THREE CASES. MOD PATHOL 2000;13:654-60.
}

To the Editor: The recent article titled "Metastasizing Fibrous Histiocytoma of the Skin: a Clinicopathologic and Immunohistochemical Analysis of Three Cases," by Guillou et al. (1), required a few comments.

Classical Virchowian pathology subdivides diseases into seven groups. Hamartomas (from Greek " $\alpha \mu \alpha \rho \tau \alpha \nu o \mu \alpha \iota$ " for "to err") define lesions, frequently congenital, sometimes developing during later life (tardive), which consist of an abnormal composition of tissue components physiologically inherent to a certain region such as nevus sebaceous or congenital melanocytic nevi. In contrast to neoplasms, their growth is not independent from, but parallels, the growth of the hosting patient or surrounding structures, respectively. Malformations are abnormal structures deriving from a misled development of the "embryonal anlage," such as craniofacial dysplasias, meningoceles, or arteriovenous shunts, and are frequently mixed up synonymously with hamartomas. Hyperplasia is an increase in number of cells (in contrast to hypertrophy, where the size of the single cell is increased, e.g., in muscular hypertrophy), which waxes and wanes with the presence of stimuli such as rubbing in prurigo nodules, human papilloma virus in warts, or iod in thyroid adenomas. Inflammatory diseases consist of an infiltrate of inflammatory cells only and, in the skin and subcutis, follow one of nine distinctive reaction patterns: superficial perivascular dermatitis; superficial and deep perivascular dermatitis; nodular and diffuse dermatitis; vasculitis; intraepidermal vesicular dermatitis; subepidermal vesicular dermatitis; folliculitis and perifolliculitis; fibrosing dermatitis; and panniculitis (2). In contrast to inflammatory diseases, neoplastic diseases are composed, at least in part, of cells other than inflammatory cells, to wit neoplastic cells. Benign and malignant neoplasms differ by their limited or unlimited autonomous growth, capable of killing the patient by local invasion or distant metastases in the latter case. Cysts are sac-like spaces filled with liquid or nonliquid substances produced by the lining epithelium. Finally, deposits are metabolic disorders that lead to the deposition of various substances within the tissue.

According to these definitions, fibrous histiocytoma is an inflammatory disease, i.e., a nodular ("granulomatous") and diffuse dermatitis in early and a fibrosing dermatitis in late stages of the process (2). The disease starts as a response to injury, which may be external, such as an insect bite or vaccination, or internal, such as a ruptured folliculitis or ruptured infundibular cyst. At the outset lesions are characterized by granulation tissue, for which these lesions were once termed sclerosing hemangiomas. As a consequence of bleeding into the lesion, numerous foam cells and siderophages can be found, a lesion then commonly known as histiocytoma. Finally, fibrosis replaces the granulomatous inflammation, the lesion shrinks and becomes increasingly hard, hence the name dermatofibroma. In time this process results in loss of fibrocytes and "homogenization" of bundles of collagen, therefore the former appellation subepidermal nodular sclerosis. Some lesions may show progressive flattening to become a hyperpigmented macule with accentuation at the periphery. At every stage this lesion is made up histopathologically of inflammatory and not neoplastic cells.

The speed, the extent, and the kind of histopathologic findings in the life cycle of dermatofibroma may vary considerably. According to these individual factors, dermatofibromas may present with a wide variety of clinicopathologic variants (3), which have been categorized (4) as follows: first, dermatofibromas with architectural peculiarities, such as deep penetrating, atrophic, or aneurysmal ("angiomatoid") variants; second, dermatofibromas with cellular/stromal peculiarities, such as epithelioid, clear cell, granular cell, atypical (pseudosarcomatous), or myxoid variants; and third, dermatofibromas with architectural and cellular/stromal peculiarities such as cellular benign fibrous histiocytoma, dermatofibroma with smooth muscle proliferation, or, most recently, combined dermatofibroma (4). Many of these variants initially had been misdiagnosed, e.g., deep penetrating dermatofibroma as dermatofibrosarcoma protuberans, aneurysmal ("angiomatoid") fibrous histiocytoma as Kaposi sarcoma, clear cell dermatofibroma as cutaneous metastasis from renal cell carcinoma, atypical (pseudosarcomatous) fibrous histiocytoma as atypical fibroxanthoma, or cellular benign fibrous histiocytoma as leiomyosarcoma, to mention but a few examples (for detailed review see Table 3 of Ref. 4). None of these lesions ever metastasized.

It is obvious that when dermatofibromas are overdiagnosed as malignancies that in reality are "benign" yet even not neoplasms, but inflammatory disorders, it is also possible vice versa. When there are metastases in "fibrous histiocytomas," as in the three cases reported by Guillou et al. (1) and five 
more cases from previous reports (5-7), the initial diagnoses have to be revised. Lesions must have been sarcomas-dermatofibrosarcoma protuberans, fibrosarcoma, and malignant fibrous histiocytoma, respectively-from the beginning, and criteria were nicely provided by Guillou et al. (1): "relatively" large size, high cellularity, marked cellular polymorphism, high mitotic activity, tumor necrosis, and repeated local recurrences. By definition, inflammatory disorders as well as benign neoplasms, which some authors (8) consider fibrous histiocytomas to be, are incapable of metastases; only malignant neoplasms can disseminate by blood and lymph vessels and across serosal surfaces.

Let's be honest! We all have gathered similar experience in daily practice. In dermatopathology the most serious error is missing a diagnosis of melanoma. Who has not had the experience of having diagnosed a Spitz nevus that later turned out to be a melanoma. Frequently, when this happens and one looks at the original specimens of the "Spitz nevus," one knows that it is a melanoma, correctly realizes the important features, and asks him- or herself, "how could I miss this melanoma?" The answer is easy: we all make mistakes, which is due to the fact that our brains do not work equally well at every time. We have to realize this and consequently say: I missed this case, let me learn something. This is much more honest, honorable, and of educational value than making bizarre diagnoses such as metastasizing fibrous histiocytoma $(1,6,7)$ or metastasizing Spitz nevus (9). I sometimes find myself asking: what would Virchow say about metastasizing benign neoplasms (or hamartomas when you regard Spitz nevus as a congenital nevus, which it frequently is) or even metastasizing inflammatory disorders. I think he would not understand it. And we would share this opinion.

Bettina G. Zelger, M.D.

Bernhard Zelger, M.D.

Departments of Pathology (BGZ) and

Dermatology (BZ)

University of Innsbruck, Innsbruck, Austria

\section{REFERENCES}

1. Guillou L, Gebhard S, Salmeron M, Coindre JM. Metastasizing fibrous histiocytoma of the skin: a clinicopathologic and immunohistochemical analysis of three cases. Mod Pathol 2000; 13:654-60.

2. Ackerman AB, Chongchitnant N, Sanchez J, Guo Y, Bennin B, Reichel M, et al., editors. Histologic diagnosis of inflammatory skin disorders. An algorithmic method based on pattern analysis. 2nd ed. Baltimore: Williams \& Wilkins; 1997.

3. Calonje E, Fletcher CDM. Cutaneous fibrohistiocytic tumors: an update. Adv Anatomic Pathol 1994;1:2-15.
4. Zelger BG, Sidoroff A, Zelger B. Combined dermatofibroma: co-existence of two or more variant patterns in a single lesion. Histopathology 2000;36:529-39.

5. Calonje E, Fletcher CDM. Aneurysmal benign fibrous histiocytoma: clinicopathological analysis of 40 cases of a tumour frequently misdiagnosed as a vascular neoplasm. Histopathology 1995;26:323-31.

6. Colome-Grimmer MI, Evans HL. Metastasizing cellular dermatofibroma. A report of two cases. Am J Surg Pathol 1996; 20:1361-7.

7. Colby TV. Metastasizing dermatofibroma. Am J Surg Pathol 1997;21:976.

8. Vanni R, Fletcher CDM, Sciot R, Dal Cin P, De Wever I, Mandahl N, et al. Cytogenetic evidence of clonality in cutaneous benign fibrous histiocytomas: a report of the CHAMP study group. Histopathology 2000;37:212-7.

9. Smith KJ, Barrett TL, Skelton HG 3rd, Lupton GP, Graham JH. Spindle cell and epithelioid cell nevi with atypia and metastases (malignant Spitz nevus). Am J Surg Pathol 1989;13:931-9.

In reply: We thank Drs. B.G. Zelger and B. Zelger for their interest in our work (1). These authors imply that all fibrous histiocytomas of the skin are inflammatory lesions, sticking in that sense to A.B. Ackerman's concept (2). We do not entirely share this view. Instead, we think that a certain proportion of fibrous histiocytomas are more likely to be tumors, whether they are located in skin, subcutis, or deep soft tissues. This view is supported by the fact that some fibrous histiocytomas (especially the cellular and aneurysmal variants) are large (over 1.5 $\mathrm{cm})$, potentially recurring lesions characterized histologically by high cellularity, marked cellular atypia, prominent mitotic activity, infiltrative borders, necrosis, and/or vascular invasion (3). Recently, clonal chromosomal abnormalities different from those observed in dermatofibrosarcoma protuberans have been demonstrated in about onethird of cutaneous fibrous histiocytomas, thus supporting the neoplastic hypothesis (4). Actually, it is our opinion that the question tumor versus reactive is of minor importance for the patient; the most important thing when facing one of those unusual variants of fibrous histiocytomas is to avoid a diagnosis of sarcoma. The purpose of our report (1) was only to validate the existence of some exceptional fibrous histiocytomas that are capable of metastatic dissemination either in lungs or regional lymph nodes, as already pointed out by other authors $(6$, 7 ), and to identify those morphologic features that could be of help in recognizing these potentially aggressive lesions. In their comment, Zelger and Zelger imply that we made a false diagnosis of fibrous histiocytoma and missed the sarcoma. They suggest we apologize for our mistakes and own up to the fact that the observations published are in fact misdiagnosed sarcomas! This is indeed not very fair, not only for the reviewers of Modern Pathology 
(who would have failed) but also for those authors who initially described this phenomenon, of whom some are known to have a strong and leader experience in soft tissue tumor pathology (5-7)! Despite the Zelger and Zelger allegations, we maintain that the lesions that we described were, morphologically and immunohistochemically, neither dermatofibrosarcomas nor fibrosarcomas or malignant fibrous histiocytomas (if the latter entity does exist as such). They showed typical features of cellular/aneurysmal fibrous histiocytomas, not different from similar lesions that have not happened to metastasize hitherto. In addition, metastatic deposits in lymph nodes were morphologically similar to primaries and did not show features of dermatofibrosarcoma or any other sarcoma. To explain this ability for some rare fibrous histiocytomas to metastasize, it is possible that these lesions gradually accumulate chromosomal/molecular aberrations as to reach a metastasizing threshold (hence deserving the term sarcoma from a biological point of view) without identifiable morphologic changes that could predict their clinical behavior. This theory also could apply to some other lesions, including lipomatous tumors, giant cell tumor of bone, inflammatory myofibroblastic tumors, and benign metastasizing leiomyomas (8). If Zelger and Zelger think that our lesions ought to be called sarcomas (as they imply), they should make a diagnosis of sarcoma on any cellular/aneurysmal fibrous histiocytoma they see in their daily practice, and, more importantly, they should treat their patients accordingly, a way of thinking and a way of doing that is totally inadequate and disproportionate in our opinion.

Although we do acknowledge the fact that, as any human being, we have made (and probably still make) misdiagnoses in our practice, there are some lesions that defy current morphological classifications, or, let's say in other words, that do not read the right books. This is probably due to the fact that, in some instances, biological events do not always convey instant (or delayed) morphological expression, as opposed to what some authors would expect.

Louis Guillou, M.D.

Sandra Gebhard, M.D.

Manuel Salmeron, M.D.

Jean-Michel Coindre, M.D.

Institutes of Pathology of Lausanne (LG, SG)

and Neuchâtel (MS)

Switzerland

Bergonié Institute and University of Bordeaux II $(J-M C)$

Bordeaux, France

\section{REFERENCES}

1. Guillou L, Gebhard S, Salmeron M, Coindre JM. Metastasizing fibrous histiocytoma of the skin: a clinicopathologic and immunohistochemical analysis of three cases. Mod Pathol 2000; 13:654-60.

2. Ackerman AB. Dermatofibroma. In: Ackerman AB, Chongchitnant N, Sanchez J, Guo Y, Bennin B, Reichel M, et al., editors. Histologic diagnosis of inflammatory skin disorders. An algorithmic method based on pattern analysis. 2nd ed. Baltimore: Williams \& Wilkins; 1997. p. 279-88.

3. Calonje E, Fletcher CDM. Cutaneous fibrohistiocytic tumors: an update. Adv Anat Pathol 1994;1:2-15.

4. Vanni R, Fletcher CDM, Sciot R, Dal Cin P, De Wever I, Mandahl N, et al. Cytogenetic evidence of clonality in cutaneous benign fibrous histiocytomas: a report of the CHAMP Study Group. Histopathology 2000;37:212-7.

5. Calonje E, Fletcher CDM. Aneurysmal benign fibrous histiocytoma: clinicopathologic analysis of 40 cases of a tumour frequently misdiagnosed as a vascular neoplasm. Histopathology 1995;26:323-31.

6. Colome-Grimmer MI, Evans HL. Metastasizing cellular dermatofibroma. A report of two cases. Am J Surg Pathol 1996; 20:1361-7.

7. Colby TV. Metastasizing dermatofibroma. Am J Surg Pathol 1997;21:976.

8. Mentzel T. Editorial. Biological continuum of benign, atypical, and malignant mesenchymal neoplasms-does it exist? J Pathol 2000;190:523-5. 